\section{Escaping abiraterone}

\section{By Lauren Martz, Staff Writer}

Although numerous papers have detailed how castration-resistant prostate cancers become refractory to Xtandi enzalutamide and other second-generation androgen receptor antagonists, comparatively little is known about how the disease eventually evades CYP17 inhibitors such as Zytiga abiraterone. Findings from a Cleveland Clinic team could change that, as the group has detailed how some tumors engage an alternative synthesis pathway for androgen. ${ }^{1}$

Androgen receptor antagonism and inhibition of CYP17 (cytochrome P450 $17 \alpha$-hydroxylase/C17, 20 lyase) are the mainstays of castrationresistant prostate cancer (CRPC) treatment. Medivation Inc. and Astellas Pharma Inc. reported $\$ 158$ million in U.S. Xtandi sales in the first half of the year, and Johnson \& Johnson's Zytiga posted sales of $\$ 739$ million in that period.

Both drugs face resistance issues, as most prostate tumors eventually bypass the testicular testosterone-dependent androgen synthesis pathway and circumvent CYP17 inhibitors by turning to adrenal androgen synthesis mechanisms (see Figure 1, "Androgen synthesis pathways in prostate cancer").

Prior studies suggested that CYP17 inhibitors may not completely shut down the CYP17-dependent pathway of androgen synthesis ${ }^{2}$ and that low levels of androgen precursors may be able to progress through the pathway.

In many tumors this should not produce enough of the androgen dihydrotestosterone (DHT) to stimulate tumor growth, but tumor growth nevertheless occurs.

In a paper published in Cell, Nima Sharifi and colleagues have solved the mystery. The team found a mutation in the hydroxysteroid $3 \beta$ dehydrogenase 1 (HSD3B1) gene encoding $3 \beta \mathrm{HSD} 1$ that catalyzes a rate-limiting step in the downstream synthesis of DHT in some CRPCs.

The mutation may be a mechanism of Zytiga drug resistance, and inhibiting its enzymatic activity could help treat CRPCs that do not respond to other therapies.

Sharifi is chair of prostate cancer research at the Cleveland Clinic. The paper also included researchers from The University of Texas Southwestern Medical Center, the University of Michigan Medical School, the University of Washington School of Medicine and the Fred Hutchinson Cancer Research Center.

The team first studied $3 \beta$ HSD 1 activity in different CRPC cell lines. Whereas one cell line converted $90 \%$ of dehydroepiandrosterone (DHEA) to androstenedione in the DHT synthesis pathway in 48 hours, the other only converted $10 \%$. Sequencing HSD3B1 in each cell line identified an $\mathrm{A}$ to $\mathrm{C}$ nucleotide substitution that resulted in an asparagine to threonine amino acid substitution in the cell line with enhanced flux.

To determine the source of the mutation, the group compared germline DNA from patients with DNA from their CRPC tumors. The team found that 3 of 25 patients who were homozygous wild-type for the gene expressed the mutation in CRPC tumor DNA. This finding suggests that the mutation can arise de novo in tumors.

However, the mutation also occurred in the germline DNA of some patients. In 3 of 11 patients who were heterozygous for the mutation, tumors evolved to express only the mutant gene. These findings suggest that screening for the HSD3B1 mutation could help identify patients unlikely to respond to available treatments.

Next, the team assessed whether the HSD3B1 mutation could be responsible for resistance to Zytiga. In surgically castrated mice with HSD3B 1 wild-type tumors, two of eight animals receiving the drug developed tumors with the mutation, whereas no tumors from vehicle-treated mice acquired the mutation.

In cultured HSD3B1-mutant CRPC cells, small hairpin RNA targeting the mutant inhibited DHEA flux and cell proliferation and decreased DHT expression compared with shRNA control. In mice with mutant tumor xenografts, shRNA targeting the mutant gene decreased tumor growth compared with shRNA control.

Finally, the team found that the mutation increased $3 \beta \mathrm{HSD} 1$ 's half-life from 2.1 to 27 hours by causing resistance to ubiquitination and degradation. These findings suggest that the mutation allows more androgen synthesis to promote tumor growth by stabilizing $3 \beta \mathrm{HSD} 1$.

\section{Drug development}

Sharifi told SciBX that his team's next step is to identify a small molecule that inhibits $3 \beta \mathrm{HSD} 1$ activity and thus cuts off the tumor's androgen supply.

Jeff Hager, senior director of biology at Seragon Pharmaceuticals Inc., said that the researchers essentially will be starting from scratch.

"One problem is that there is no proof-of-concept compound with good drug-like properties that could selectively inhibit the enzyme," Hager said. "They need to determine first how druggable this enzyme is, and ideally they should look for a nonsteroidal small molecule inhibitor with improved bioavailability over steroidal compounds."

Hager said that abiraterone could alternatively be used as a starting point for medicinal chemistry in the steroid scaffold. According to the authors of the paper, the drug weakly inhibits $3 \beta$ HSD 1 . However, Hager cautioned that "hormonal synthesis is a complex process, and there 


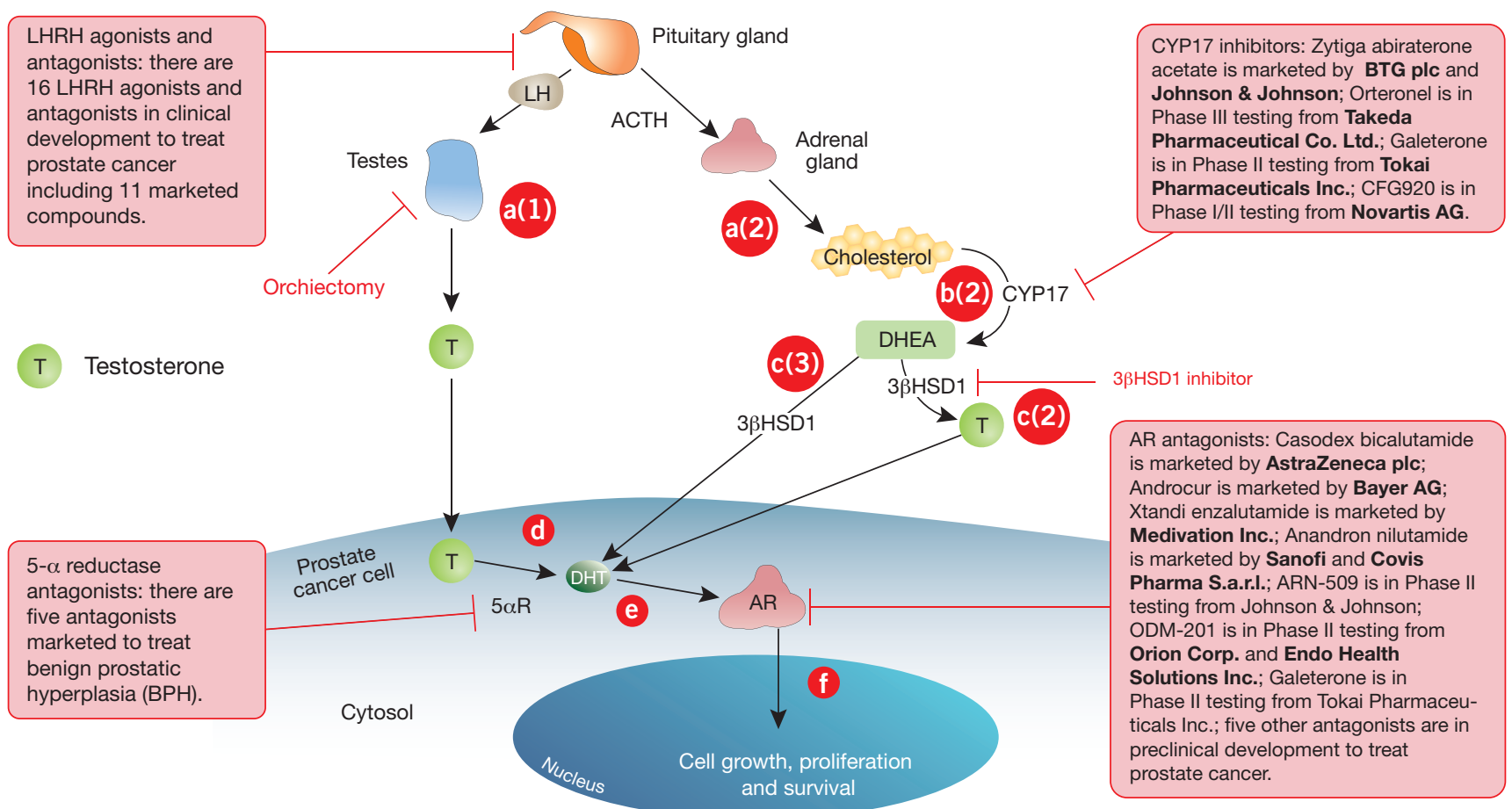

Figure 1. Androgen synthesis pathways in prostate cancer. In testicular androgen synthesis, luteinizing hormone-releasing hormone $(\mathrm{LHRH})$ stimulates release of luteinizing hormone $(\mathrm{LH})$ from the pituitary gland, which signals the testes to secrete testosterone [a(1)]. Testosterone enters the prostate cancer cell and is converted to the potent androgen dihydrotestosterone (DHT) by 5- $\alpha$ reductase (5 $\alpha \mathrm{R})$ [d]. DHT binds and activates the androgen receptor (AR) [e], which in turn initiates transcription of oncogenic genes increasing cell growth, proliferation and survival [f].

In castration-resistant androgen synthesis, the pituitary gland also signals the adrenal gland to initiate an adrenocorticotropic hormone (ACTH)-mediated androgen synthesis pathway $[\mathbf{a}(2)]$. The switch from dependence on the testicular to the adrenal androgen synthesis pathway can occur following surgical or chemical castration, leading to tumor regrowth. Through a series of reactions catalyzed by cytochrome P450 $17 \alpha$-hydroxylase/17, 20 lyase (CYP17), cholesterol is converted into dehydroepiandrosterone (DHEA) [b(2)].The CYP17 inhibitor abiraterone blocks the production of DHEA and downstream androgen synthesis in the adrenal androgen synthesis pathway. However, it may incompletely inhibit this step and lead to resistance. Hydroxysteroid $3 \beta$ dehydrogenase 1 (HSD3B1), the gene that encodes 3 $\beta$ HSD1, catalyzes the rate-limiting step in the pathway that converts DHEA to testosterone, which is then converted to DHT to activate AR [c(2)]. DHEA may also be converted to DHT independently of testosterone through a 3 $\beta$ HSD1-dependent pathway [c(3)]. A gain-of-stabilization mutation in HSD3B1 can increase the flux from DHEA to DHT, possibly allowing sufficient production of DHT from the residual androgen precursors remaining during abiraterone treatment leading to therapeutic resistance. Inhibitors of 3bHDS1 may prevent this resistance pathway.

the consequences of $3 \beta$ HSD1 inhibition that may occur in humans. Blocking $3 \beta$ HSD1 will inhibit not only the catalytic step converting DHEA to androstenedione but also the conversion of pregnenolone or 17-hydroxyprenenolone to steroid metabolites such as progesterone, deoxycorticosterone, corticosterone and cortisol."

As a result, he said, it will be necessary "to strike a balance between $3 \beta \mathrm{HSD} 1$ inhibition and other unwanted consequences resulting from $3 \beta$ HSD1 blockade. This can be determined by examining the toxicology of novel 3ßHSD1 inhibitors."

Tokai's Galeterone, a small molecule that disrupts androgen receptor signaling through a triple mechanism that involves antagonizing testosterone binding to the androgen receptor, inhibiting CYP17 and degrading the androgen receptor, is in Phase II testing for CRPC.

Seragon is developing selective estrogen receptor degraders including ARN-810, which is in Phase I testing to treat estrogen receptor-positive metastatic breast cancer. Aragon Pharmaceuticals Inc. spun out Seragon prior to Aragon's acquisition by J\&J in September.

Aragon's second-generation androgen receptor antagonist, ARN-509, is in Phase II testing to treat CRPC. Hager was senior director of biology at Aragon.

\section{Added benefits}

If the Cleveland Clinic generates an inhibitor of 3 $\beta$ HSD1, the molecule would likely find immediate use as part of a combination therapy for CRPC.

Hager told SciBX, "A real advantage of this potential new target is that it adds another layer to the antiandrogen therapeutic options. Each level of therapy lowers androgen levels, and adding a $3 \beta \mathrm{HSD} 1$ inhibitor could shut down the androgen supply more completely. One could imagine a combination of LHRH [luteinizing hormone-releasing hormone] agonists to block testicular androgen, CYP17 inhibitors to block adrenal androgen and a new $3 \beta$ HSD1-targeting agent to block the amplified DHT production that the mutation confers. If the combination 
are a lot of related enzymes and compensatory feedback. An inhibitor that is not sufficiently selective could affect other important biological processes such as aldosterone production and blood pressure."

But Douglas Jacoby, head of research at Tokai Pharmaceuticals Inc., worried that "the mouse model does not accurately recapitulate

\section{"Based on currently}

available data, the 3ßHSD1 pathway appears to be important in a minority of patients. The mutational status of 3BHSD1 could serve as a biomarker to identify those patients who are likely to benefit from this mode of therapy." -William Olson, Progenics Pharmaceuticals Inc. the consequences of $3 \beta$ HSD 1 inhibition that may occur in humans. Blocking $3 \beta$ HSD 1 will inhibit not only the catalytic step converting DHEA to androstenedione but also the conversion of pregnenolone or 17-hydroxyprenenolone to steroid metabolites such as progesterone, deoxycorticosterone, corticosterone and cortisol."

As a result, he said, it will be necessary "to strike a balance between $3 \beta \mathrm{HSD} 1$ inhibition and other unwanted consequences resulting from $3 \beta \mathrm{HSD} 1$ blockade. This can be determined by examining the toxicology of novel 3ßHSD1 inhibitors."

Tokai's Galeterone, a small molecule that disrupts androgen receptor signaling through a triple mechanism that involves antagonizing testosterone binding to the androgen receptor, inhibiting CYP17 and degrading the androgen receptor, is in Phase II testing for CRPC.

Seragon is developing selective estrogen receptor degraders including ARN-810, which is in Phase I testing to treat estrogen receptor-positive metastatic breast cancer. Aragon Pharmaceuticals Inc. spun out Seragon prior to Aragon's acquisition by J\&J in September.

Aragon's second-generation androgen receptor antagonist, ARN-509, is in Phase II testing to treat CRPC. Hager was senior director of biology at Aragon.

\section{Added benefits}

If the Cleveland Clinic generates an inhibitor of 3 3 HSD1, the molecule would likely find immediate use as part of a combination therapy for CRPC.

Hager told SciBX, "A real advantage of this potential new target is that it adds another layer to the antiandrogen therapeutic options. Each level of therapy lowers androgen levels, and adding a 3 $3 \mathrm{HSD} 1$ inhibitor could shut down the androgen supply more completely. One could imagine a combination of LHRH [luteinizing hormone-releasing hormone] agonists to block testicular androgen, CYP17 inhibitors to block adrenal androgen and a new 3 $\beta$ HSD1-targeting agent to block the amplified DHT production that the mutation confers. If the combination is possible from a tolerability perspective, one could imagine shutting down the hormone production more completely."

William Olson, SVP of R\&D at Progenics Pharmaceuticals Inc., added that $3 \beta$ HSD1 inhibition might not only treat resistant tumors but also prevent resistance in the first place by blocking the alternative androgen source before tumors evolve to depend on it.

Progenics' PSMA ADC, an antibody-drug conjugate targeting prostatespecific membrane antigen (PSMA; FOLH1; GCPII), is in Phase II testing to treat prostate cancer.
Hager added, “There are non-overlapping mechanisms of acquired drug resistance, and altering the drug cocktail to better target the mutations and genetics of different tumors can all be complementary, especially when coupled with predictive biomarkers. These drugs all may have their roles."

\section{Companionship}

Olson said that the number of patients likely to benefit from $3 \beta$ HSD 1 inhibition might be small. "Based on currently available data, the $3 \beta$ HSD 1 pathway appears to be important in a minority of patients. The mutational status of $3 \beta \mathrm{HSD} 1$ could serve as a biomarker to identify those patients who are likely to benefit from this mode of therapy," he said.

But Sharifi said that the data in the paper suggest that about $20 \%$ of patients with CRPC may have either the germline mutation with loss of heterozygosity or a somatic de novo mutation in the enzyme.

"Our data probably underrepresent the true frequency of the mutation because some of the patients we studied were progressing but some were from an autopsy population. If we are looking specifically at progressing tumors, the frequency might be higher," he said.

Whatever the exact number, Hager said that given the size of the CRPC market, "north of $5 \%-10 \%$ affected is a reasonable opportunity and market size."

Sharifi said that his team's next step for biomarker development is to link the HSD3B1 biomarker to clinical trials for various types of hormone therapy to see if it predicts response.

Hager said, "Such a biomarker test could have broad importance because it could help select patients who produce high DHT levels. Whether these patients are specifically resistant to abiraterone or not, this indicates that the tumors likely still depend on androgens, which is valuable information to help guide treatment decisions. The potential to develop a biomarker test is important because it suggests an almost immediate application for the findings."

Sharifi told SciBX that the Cleveland Clinic has filed two provisional patent applications covering the work that are available for licensing.

Martz, L. SciBX 6(38); doi:10.1038/scibx.2013.1049

Published online Oct. 3, 2013

\section{REFERENCES}

1. Chang, K.-H. et al. Cell; published online Aug. 29, 2013; doi:10.1016/j.cell.2013.07.029

Contact: Nima Sharifi, Cleveland Clinic, Cleveland, Ohio e-mail: sharifn@ccf.org

2. Attard, G. et al. J. Clin. Endocrinol. Metab. 97, 507-516 (2012)

COMPANIES AND INSTITUTIONS MENTIONED

Aragon Pharmaceuticals Inc., San Diego, Calif.

Astellas Pharma Inc. (Tokyo:4503), Tokyo, Japan

Cleveland Clinic, Cleveland, Ohio

Fred Hutchinson Cancer Research Center, Seattle, Wash.

Johnson \& Johnson (NYSE:JNJ), New Brunswick, N.J.

Medivation Inc. (NASDAQ:MDVN), San Francisco, Calif.

Progenics Pharmaceuticals Inc. (NASDAQ:PGNX), Tarrytown, N.Y.

Seragon Pharmaceuticals Inc., San Diego, Calif.

Tokai Pharmaceuticals Inc., Cambridge, Mass.

University of Michigan Medical School, Ann Arbor, Mich.

The University of Texas Southwest Medical Center, Dallas, Texas

University of Washington School of Medicine, Seattle, Wash. 